\title{
Guidewire localization with transesophageal echocardiography: do not forget the left side
}

\author{
José Luis Carrasco Del Castillo, MD • \\ Jean Bussières, MD • Antoine G. Rochon, MD • \\ André Y. Denault, MD, PhD
}

Received: 22 December 2011/Accepted: 30 April 2012/Published online: 30 May 2012

(C) Canadian Anesthesiologists' Society 2012

\section{To the Editor,}

To avoid unintended arterial puncture when using a Seldinger technique, surface ultrasound may be a valuable tool to help confirm proper correct positioning of a guidewire within the internal jugular vein. ${ }^{1}$ However, the use of ultrasound for this indication has several limitations. It is not always possible to exclude the possibility of carotid artery puncture, and ultrasonography does not allow for complete visualization of the guidewire advancing into the superior vena cava (SVC). Blaivas ${ }^{2}$ has reported cases of unintended arterial cannulation despite the use of ultrasound guidance. To exclude possible arterial puncture, manometry, continuous electrocardiogram monitoring, and arterial blood gas analysis can still be used. ${ }^{3}$

In cases where transesophageal echocardiography (TEE) is being used, TEE can follow the progression of the guidewire into the SVC prior to introducing the dilators, introducer sheath, or catheter into the internal jugular vein. ${ }^{4}$ The TEE images are used to verify, in real time, the progression of the guidewire from the SVC to the right atrium by using the mid-esophageal (ME) bicaval view $90-110^{\circ}{ }^{4}$ When the guidewire is not observed in either the SVC or the right atrium, turning the transesophageal ultrasound probe to the left and looking for the aortic root

J. L. Carrasco Del Castillo, MD · J. Bussières, MD $(\square)$

Institut universitaire de cardiologie et pneumologie,

Université Laval, Québec, QC, Canada

e-mail: jbuss@criucpq.ulaval.ca

A. G. Rochon, MD - A. Y. Denault, MD, PhD

Institut de Cardiologie de Montréal, Montréal, QC, Canada
(ME long-axis ascending aorta view) (Figure), the aortic arch (upper esophageal aortic arch short-axis $0^{\circ}$ and longaxis view $90^{\circ}$ ), or the descending aorta (descending aorta short-axis $0^{\circ}$ and long axis-view $90^{\circ}$ ) may help to localize the guidewire. If the guidewire is not sited within the SVC or major arteries, we suggest expanding the ultrasound field to examine the subclavian veins, the contralateral internal or external jugular veins, or ruling out possible persistent left SVC. Finally, if necessary, manometry may be used to distinguish venous from arterial cannulation.

In conclusion, TEE may be an important tool to verify correct guidewire positioning during central venous cannulation. If the guidewire is not visualized within the SVC during TEE examination, it is important to search for and exclude arterial siting. It may be important to turn the TEE probe to the left to facilitate optimal visualization.

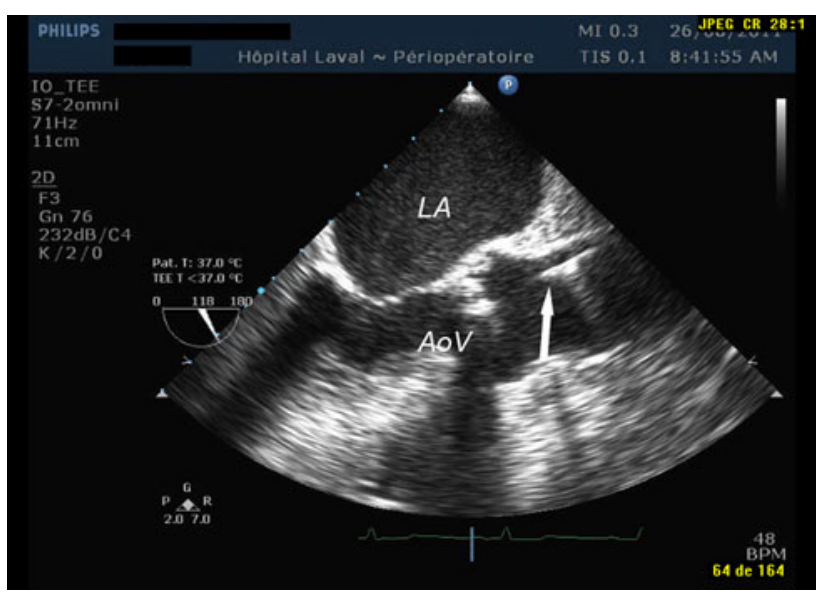

Figure Mid-esophageal long-axis ascending aorta view showing a cannulation guidewire inside the ascending aorta (arrow). LA $=$ left atrium; AoV = aortic valve 
Competing interests None declared.

\section{References}

1. Troianos CA, Hartman GS, Glas KE, et al. Guidelines for performing ultrasound guided vascular cannulation: recommendations of the American Society of Echocardiography and the Society of Cardiovascular Anesthesiologists. J Am Soc Echocardiogr 2011; 24: 1291-318.
2. Blaivas M. Video analysis of accidental arterial cannulation with dynamic ultrasound guidance for central venous access. J Ultrasound Med 2009; 28: 1239-44.

3. Rupp SM, Apfelbaum JL, Blitt C, et al. Practice guidelines for central venous access: a report by the American Society of Anesthesiologists Task Force on Central Venous Access. Anesthesiology 2012; 116: 539-73.

4. Sawchuk C, Fayad A. Confirmation of internal jugular guide wire position utilizing transesophageal echocardiography. Can J Anesth 2001; 48: 688-90. 